\title{
Avaliação dos pés em pessoas com diabetes mellitus hospitalizadas
}

\section{Evaluation of feet in people with diabetes mellitus hospitalized}

\author{
Luciana Martins da Rosa' • Melissa Orlandi Honório Locks² ${ }^{2}$ Laura Cavalcanti de Farias Brehmer ${ }^{3}$ \\ Bruna Pedroso Canever ${ }^{4}$ Gisele Cristina Manfrini ${ }^{5}$ Emanuele Pozzebon Caurio ${ }^{6}$
}

\begin{abstract}
RESUMO
Objetivo: Analisar as características dos pés em pessoas com diabetes mellitus. Método: Estudo observacional descritivo realizado em um hospital escola. Foram incluídas 38 pessoas hospitalizadas com diagnóstico de diabetes. A coleta de dados ocorreu entre agosto e dezembro de 2017, durante consulta de enfermagem à beira do leito, que incluiu questionário semi -estruturado relacionadas à avaliação dos pés. Os dados coletados foram submetidos à estatística descritiva simples e foram apresentados na forma descritiva e em tabelas. Resultados: $50 \%$ dos participantes de cada sexo; $89,47 \%$ com diabetes tipo 2 e com média de idade de 60,29 anos; 52,62\% com baixa escolaridade (sem instrução e com fundamental incompleto); $71 \%$ desconheciam a temática pé diabético; $68,42 \%$ nunca haviam realizado avaliação dos pés com profissional. Na classificação de risco 26,31\% dos participantes apresentaram risco entre I-3 para pé diabético. Conclusão: Os resultados deste estudo evidenciam que grande parte dos pacientes, apesar de terem o diagnóstico da doença por longo tempo, ainda desconhecem cuidados essenciais com os pés, realizam práticas inadequadas e raramente recebem orientações específicas para prevenir e evitar lesões nos pés.
\end{abstract}

Descritores: Diabetes mellitus; Complicações do Diabetes; Pé diabético; Enfermagem; Cuidado de Enfermagem.

\begin{abstract}
Objective:To analyze the characteristics of the feet in people with diabetes mellitus. Method: Observational descriptive study carried out in a School Hospital.Were participants 38 people hospitalized diagnosed with diabetes. Data collection took place between August and December 2017, during a nursing consultation at the bedside, which included a semi-structured questionnaire related to foot evaluation. The collected data were submitted to simple descriptive statistics and were presented in descriptive form and in tables. This article presents the collected variables related to the evaluation of the feet. Results: $50 \%$ of participants of each sex; $89.47 \%$ with type 2 diabetes and with an average age of 60.29 years; $52.62 \%$ with low education (without education and with incomplete elementary); $71 \%$ were unaware of the diabetic foot theme; $68.42 \%$ had never performed a professional foot evaluation. In the risk classification, $26.3 \mathrm{l} \%$ of the participants had a risk of I-3 for diabetic foot. Conclusion:The results of this study show that most patients, despite having been diagnosed with the disease for a long time, are still unaware of essential foot care, perform inadequate practices and rarely receive specific guidance to prevent and avoid foot injuries.
\end{abstract}

Descriptors: Diabetes Mellitus; Diabetes Complications; Diabetic Foot; Nursing. Nursing Care.

I Enfermeira. Professora do Departamento de Enfermagem e do Programa de Pós-Graduação Gestão do Cuidado em Enfermagem da Universidade Federal de Santa Catarina.Vice Líder do Laboratório de Tecnologia, Pesquisa e Inovação Cuidando \& Confortando. Florianópolis, Santa Catarina, Brasil. Orcid: https://orcid.org/0000$0002-1884-5330$

2 Enfermeira. Professora do Departamento de Enfermagem e do Programa de Pós-Graduação Gestão do Cuidado em Enfermagem da Universidade Federal de Santa Catarina. Pesquisadora do Laboratório de Pesquisas e Tecnologias em Enfermagem, Cuidado em Saúde a Pessoas Idosas.. Florianópolis, Santa Catarina, Brasil.Orcid: https://orcid.org/0000-0003-0972-2053

3 Enfermeira. Professora do Departamento de Enfermagem e do Programa de Pós-Graduação em Enfermagem da Universidade Federal de Santa Catarina.Vice Líder do Laboratório de Pesquisas sobre Trabalho, Ética, Saúde e Enfermagem. Florianópolis, Santa Catarina, Brasil. Orcid: https://orcid.org/0000-000I-9965-88I I

4 Professora do Departamento de Enfermagem da Universidade Federal de Santa Catarina.Vice Líder do Laboratório de Pesquisa e Tecnologia em Educação em Enfermagem e Saúde. Florianópolis, Santa Catarina, Brasil. Orcid: https://orcid.org/0000-0002-3484-0740

5 Enfermeira. Professora do Departamento de Enfermagem e do Programa de Pós-Graduação em Enfermagem da Universidade Federal de Santa Catarina.Vice Líder do Laboratório de Pesquisa em Enfermagem e Promoção da Saúde. Florianópolis, Santa Catarina, Brasil. Orcid: https://orcid.org/0000-0003-0445-1610

6 Acadêmica de Enfermagem da Universidade Federal de Santa Catarina. Bolsista PróBolsas/2019, Pró Reitoria de Extensão. Florianópolis, Santa Catarina, Brasil. Orcid:https://orcid.org/0000-0002-7365-1336 


\section{INTRODUÇÃO}

As neuropatias diabéticas se caracterizam pela perda progressiva das fibras nervosas e representam um grupo de doenças decorrentes de complicações crônicas do Diabetes mellitus (DM). Neste conjunto se inclui a polineuropatia diabética sensitivo-motora crônica ou neuropatia diabética periférica fortemente associada ao desenvolvimento de úlceras nos pés de pessoas com diabetes. $O$ conceito de pé diábetico relaciona-se à neuropatia diabética periférica, além de agregar outros elementos, ou não, como infecção, ulceração e destruição de tecidos profundos associados à anormalidades neurológicas e à doença arterial periférica dos membros inferiores ${ }^{(1)}$.

A doença do pé afeta quase $6 \%$ das pessoas com diabetes e inclui infecção, ulceração ou destruição dos tecidos do pé. Pode prejudicar a qualidade de vida dos pacientes e afetar o convívio social e os meios de subsistência. Entre $0,03 \%$ e I,5\% dos pacientes com pé diabético necessitarão de uma amputação. A maioria das amputações começa com úlceras e podem ser evitadas com o adequado cuidados com os pés e rastreamento para avaliar o risco de complicações nos pés ${ }^{(2-4)}$.

O pé diabético se associa à significativas taxas de mortalidade e a custos significativos para o sistema público de saúde. Este fato evidencia a necessidade de políticas de saúde voltadas para a proteção da saúde e melhorias da qualidade da atenção à pessoas com diabetes na Rede de Assistencia à Saúde ${ }^{(5)}$.

A prevenção das conplicações do diabetes começa por um bom controle glicêmico, especificamente, para a prevenção do pé diabético recomenda-se a avaliação regular dos pés, o uso de calçados adequados, a educação do paciente e o tratamento precoce das lesões pré -ulcerativas. A ulceração nos pés quando presente, com sinais de infecção, sepse ou isquemia exige atendimento em centro especializado, com tratamento cirúrgico, de revascularização e de reabilitação. $A$ avaliação regular dos pés em pessoas com diabetes com baixo risco para 0 pé diabético deve ocorrer anualmente. A cada avaliação deve ser enfatizada a importância dos cuidados com os pés e controle glicêmico. Para os pacientes com risco moderado ou alto, como aqueles com uma deformidade no pé ou com diagnóstico de neuropatia periférica ou doença arterial periférica, recomenda-se acompanhamento mais frequente; a periodicidade dependerá da condição do acometimento ${ }^{(4)}$.

A educação em saúde das pessoas com diabetes, de maneira estruturada, organizada e contínua, combinada com intervenções preventivas, pode prevenir problemas nos pés. Contudo, ações nesta perspectiva são complexas, mesmo diante das dificuldades recomenda-se a orientação das pessoas com diabetes para melhorar o conhecimento e o comportamento do cuidado com os pés ${ }^{(6-7)}$.
Por intervenção preventiva destacam-se a consulta de enfermagem e outros produtos de enfermagem, promotores do cuidado aliado à educação em saúde. Como exemplo de produto citam-se os manuais educativos produzidos por enfermeiros, uma tecnologia de enfermagem que retrata o comprometimento do enfermeiro com a qualidade da assistência prestada para melhor qualidade de vida da pessoa cuidada, neste caso pessoas com diabetes ${ }^{(8)}$.

Considerando a epidemiologia do diabetes no Brasil, 26 milhões de casos no ano de $2017^{(9)}$, o número de pessoas com diabetes com pé diabético e a necessidade de educação em saúde para este grupo de atenção, desenvolveu-se um projeto de extensão universitária, tendo como foco da atenção a educação em saúde de pessoas com diabetes. Para o alcance da educação em saúde, considerando sua abrangência, realizou-se consulta de enfermagem a beira do leito. Desta experiência emergiu a seguinte questão norteadora "qual o perfil e quais os cuidados com os pés de pessoas com diabetes mellitus hospitalizadas"? Portanto, o objetivo deste estudo é identificar as características e os cuidados dos pés em pessoas com diabetes mellitus hospitalizadas.

\section{MÉTODO}

Trata-se de um estudo observacional descritivo, com abordagem quantitativa, realizado em um hospital escola do Sul do Brasil. A população do estudo constitui-se de pessoas com DM tipo I ou 2, internadas nas unidades de clínicas cirúrgicas e médicas do cenário do estudo, no período entre agosto e dezembro de 20 I7.A amostra do estudo foi não probabilística, por conveniência.

Foram considerados critérios de inclusão a registro do diagnóstico de DM no prontuário do paciente e um período de internação superior a dois dias, com estado de saúde, condição cognitiva que permitisse aceitar participar da pesquisa e responder ao questionário. Foram incluídas no estudo 38 pessoas.

A coleta dos dados ocorreu através de questionário semiestruturado composto de duas partes, uma contendo a caracterização do participante e a outra incluindo informações específicas acerca da avaliação dos pés.

As variáveis definidas para este estudo foram: diagnóstico de diabetes (tipo); idade; sexo; escolaridade; tempo de diagnóstico do diabetes; conhecimento sobre pé diabético; avaliação prévia dos pés por profissional da saúde; tempo de realização da última avaliação dos pés; cuidados com os pés (hidratada, hábito de realização de escalda pés; pessoa que realiza o corte das unhas dos pés; instrumento utilizado para corte das unhas; corte da unha no momento da coleta dos dados (adequado ou inadequado); hábito de ficar des- 
calço; tipo de calçado utilizado; horário para compra dos sapatos); sensação de queimação nos pés; dor ao calçar os sapatos; dor nos pés; sensação de agulhadas nos pés; presença de câimbras; presença de formigamentos e dormência; presença de alterações anatômicas e da pele dos pés; alteração da sensibilidade protetora plantar (com aplicação de monofilamento) e vibratória (com aplicação de diapasão); classificação de risco para o pé diabético. Registra-se que a avaliação dos pés foi realizada segundo a recomendação da Sociedade Brasileira de Diabetes ${ }^{(10)}$.

Os dados coletados foram submetidos à estatística descritiva simples e foram apresentados na forma descritiva e em tabelas. A discussão dos dados foi realizada a partir das publicações científicas atualizadas sobre o tema do estudo.

Este estudo atendeu às recomendações da Resolução n. ${ }^{\circ} 466 /$ I 2 do Conselho Nacional de Saúde para pesquisa com seres humanos e foi aprovado pelo Comitê de Ética da Instituição sob parecer Certificado de Apresentação e Apreciação Ética 69305317.0.0000.0121.

\section{RESULTADOS}

Foram incluídos no estudo 38 (100\%) pessoas com
DM, sendo 19 (50\%) do sexo masculino e $19(50 \%)$ do sexo feminino.

Quanto à escolaridade, quatro (10,52\%) participantes não tinham instrução, $16(42,10 \%)$ possuíam o ensino fundamental incompleto, quatro $(10,52 \%)$ ensino fundamental completo, três $(7,92 \%)$ ensino médio incompleto, dez $(26,31 \%)$ médio completo e um $(2,63 \%)$ ensino superior completo.

Em relação aos diagnósticos médicos, quatro (10,52\%) participantes tinham diabetes tipo I, com tempo de diagnóstico oscilando entre I e 49 anos, idade mínima de 49 e máxima de 68 anos, média 60,29 anos. Quanto aos pacientes com diabetes tipo 2 foram contabilizados 34 $(89,47 \%)$, com tempo de diagnóstico oscilando entre menos de um ano e 44 anos, idade mínima de 28 anos e máxima de 74 anos, média 60,29 anos.

$\mathrm{Na}$ avaliação quanto às características dos membros inferiores a totalidade das alterações encontradas é apresentada na tabela I.

Ao associar os dados oriundos do exame físico e dos testes específicos para avaliação da sensibilidade, procedeu-se a classificação dos pacientes para o risco do pé diabético.A maioria $(73,68 \%)$ dos participantes foi classificada com o risco 0 . Os demais scores são descritos na tabela 2 .

TABELA 1 - Alterações encontradas nos pés direito e esquerdo, segundo avaliação de risco do pé diabético. Florianópolis, SC, Brasil, 2017

\begin{tabular}{|c|c|c|}
\hline Variáveis & $\begin{array}{c}\text { Pé direito } \\
n(\%)\end{array}$ & $\begin{array}{c}\text { Pé esquerdo } \\
n(\%)\end{array}$ \\
\hline Dedos em garra & $4(10,53)$ & $5(13,16)$ \\
\hline Dedos em sobreposição & $1(2,63)$ & -- \\
\hline $\begin{array}{l}\text { Lesões } \\
\qquad \begin{array}{l}\text { Úlcera no calcâneo } \\
\text { Gangrena } \\
\text { Amputações de Pododáctilos } \\
\text { Lesões esfoliativas }\end{array}\end{array}$ & $\begin{array}{c}1(2,63) \\
1(2,63) \\
1(2,63) \\
17(44,74)\end{array}$ & $\begin{array}{c}-- \\
1(2,63) \\
1(2,63) \\
16(42,11)\end{array}$ \\
\hline $\begin{array}{l}\text { Alterações na pele } \\
\text { Xerose } \\
\text { Palidez } \\
\text { Fria } \\
\text { Vermelhidão } \\
\text { Cianose }\end{array}$ & $\begin{array}{l}18(47,37) \\
12(31,58) \\
4(10,53) \\
6(15,79) \\
3(7,92)\end{array}$ & $\begin{array}{l}18(47,37) \\
12(31,58) \\
5(13,16) \\
7(18,42) \\
3(7,92)\end{array}$ \\
\hline Rarefação dos pelos das pernas & $20(52,63)$ & $21(55,63)$ \\
\hline Calosidades & $11(28,95)$ & $8(21,50)$ \\
\hline $\begin{array}{l}\text { Corte da unha } \\
\text { Adequado } \\
\text { Inadequado }\end{array}$ & $\begin{array}{l}26(68,42) \\
12(31,58)\end{array}$ & $\begin{array}{l}26(68,42) \\
12(31,58)\end{array}$ \\
\hline $\begin{array}{l}\text { Sensibilidade protetora plantar } \\
\text { Sensibilidade presente } \\
\text { Sensibilidade alterada }\end{array}$ & $\begin{array}{l}28(73,68) \\
10(26,32)\end{array}$ & $\begin{array}{c}31(81,58) \\
7(18,42)\end{array}$ \\
\hline $\begin{array}{l}\text { Sensibilidade vibratória } \\
\text { Sensibilidade presente } \\
\text { Sensibilidade ausente } \\
\text { Sensibilidade diminuída }\end{array}$ & $32(84,21) 5(13,16) 1(2,63)$ & $\begin{array}{c}35(92,11) \\
2(5,26) \\
1(2,63)\end{array}$ \\
\hline
\end{tabular}


Em relação ao conhecimento dos participantes sobre pé diabético, 27 (7I,05\%) apontaram nunca ter ouvido falar sobre o tema, dos que tinham conhecimento, cinco (13,16\%) foram informados por profissional da área da saúde; 26 (68,42\%) nunca passaram por avaliação dos pés por profissional de saúde, dos que foram submetidos à avaliação dos pés, quatro $(10,53 \%)$ a realizaram nos últimos seis meses, três $(7,92 \%)$ no último ano, um $(2,63 \%)$ há 15 anos e três não recordaram (7,92\%).

Dentre os resultados relacionados aos cuidados com os pés a tabela 3 apresenta os achados segundo as variáveis questionadas.

Em relação à alteração de percepção dos pés, dez participantes $(26,31 \%)$ referiram sentir queimação, nove (26,68\%) dor ao calçar sapatos, 18 (47,36\%) dor intermitente, $12(31,57 \%)$ agulhadas, $20(52,63 \%)$ câimbras, 15 $(39,47 \%)$ dormência e 15 (39,47\%) formigamentos.

\section{DISCUSSÃO}

O Brasil ocupa a $4^{a}$. posição entre os países que possuem mais casos de diabetes, chegando a I4,3 milhões em $2015^{(9)}$. Em pesquisa realizada pelo Instituto Brasileiro de Geografia e Estatística identificou-se que do total da população, cerca de 7\% eram de mulheres e 5,4 homens, sendo que a maior taxa foi identificada na parcela da população com baixa escolaridade ${ }^{(11)}$. No estudo em questão corrobora-se com esses aspectos epidemiológicos, considerando-se que a maior parte dos participantes possuía ensino fundamental incompleto, entretanto, no que se refere ao sexo, identificou-se paridade entre mulheres e homens.

No que se refere à classificação do DM, a mesmo tem sido definida segundo sua etiologia. O DM tipo I, apesar de estar apresentando uma prevalência de crescimento intensificado nas últimas décadas, atinge ainda a minoria da

TABELA 2 - Classificação de risco do pé diabético. Florianópolis, SC, Brasil, 2017

\begin{tabular}{c|c}
\hline Classificação de risco & $\mathrm{n}(\%)$ \\
\hline Risco 0 & $28(73,68)$ \\
Risco 1 & $7(18,42)$ \\
Risco 2 & $2(5,26)$ \\
Risco 3 & $1(2,63)$ \\
\hline Total & $38(100)$ \\
\hline
\end{tabular}

TABELA 3 - Cuidados com os pés. Florianópolis, SC, Brasil, 2017

\begin{tabular}{|c|c|}
\hline Cuidados & $\mathrm{n}(\%)$ \\
\hline \multicolumn{2}{|l|}{ Hidratação dos pés } \\
\hline Duas vezes na semana & $10(26,32)$ \\
\hline Três vezes na semana & $5(13,16)$ \\
\hline Uma vez na semana & $3(7,92)$ \\
\hline A cada 15 dias & $3(7,92)$ \\
\hline Ausência de secagem dos pés após o banho & $27(71,05)$ \\
\hline Realização de escalda pés & $18(47,37)$ \\
\hline \multicolumn{2}{|l|}{ Corte das unhas } \\
\hline Pela própria pessoa com diabetes & $17(44,74 \%)$ \\
\hline Por familiares & $16(42,11 \%)$ \\
\hline Por pedicure & $5(13,16)$ \\
\hline \multicolumn{2}{|l|}{ Instrumento utilizado para corte das unhas } \\
\hline Cortador de unhas & $18(47,37)$ \\
\hline Tesoura & $10(26,32)$ \\
\hline Alicate de unhas & $9(23,68)$ \\
\hline Lixa & $1(2,63)$ \\
\hline \multicolumn{2}{|l|}{ Tipo de sapatos utilizados } \\
\hline Sandálias de tiras & $18(47,37)$ \\
\hline Tênis & $9(23,68)$ \\
\hline Sapatos fechados arredondados (no bico) & $1(2,63)$ \\
\hline Sapatos linha confort & $1(2,63)$ \\
\hline Outros sapatos & $9(23,68)$ \\
\hline \multicolumn{2}{|l|}{ Horário para compra dos sapatos } \\
\hline Sem preocupação com horário para compra & $20(53,63)$ \\
\hline Desconheciam a importância do horário para compra dos sapatos & $6(15,79)$ \\
\hline Período da manhã & $3(7,92)$ \\
\hline Período da tarde & $7(18,42)$ \\
\hline Período da noite & $2(5,26)$ \\
\hline
\end{tabular}


população, ficando entre 5 e $10 \%$ dos casos, prevalência também encontrada nesse estudo (10,5\%).Ao passo em que os casos de DM tipo 2 desta pesquisa também são similares aos da literatura, apontando incidência de cerca $90 \%(10)$.

Em relação às características avaliadas nos pés, identificou-se que as alterações detectadas entre os pés direito e esquerdo são semelhantes, destacando-se as lesões esfoliativas, ressecadas e calosidades. Uma pesquisa com variáveis semelhantes, no contexto da Atenção Primária à Saúde, também evidenciou prevalência de alterações nas condições dermatológicas e estruturais de pessoas com DM(12).

As alterações de pele, sobretudo a xerose e as calosidades são achados frequentemente em estudos com pessoas com diabetes ${ }^{(13-14)}$. As manifestações dermatológicas devem ser investigadas e tratadas, pois se constituem em lesões pré-ulcerativas decorrentes de Polineuropatia Diabética e Doença Arterial Periférica. Especificamente as calosidades que se trata de um espessamento epidérmico causado por traumatismos locais recorrentes, são mais evidentes em áreas de alta pressão, como exemplo da região plantar ${ }^{(15)}$.

Todas as alterações descritas são importantes de serem observadas, acompanhadas e tratadas sempre que necessário. A falta de cuidados com estas alterações e a manipulação inadequada das mesmas levam ao agravamento do quadro podendo chegar às amputações. Quanto maior o tempo de ocorrência dos problemas relacionados com os pés, dificuldades de acesso a exames e desinformação sobre cuidados com os pés são fatores associados às amputações ${ }^{(16)}$.

Acerca do conhecimento sobre o pé diabético, constatou-se que a maioria nunca havia ouvido falar sobre o tema, bem como, nunca tivera seus pés examinados, assim, identificou-se uma necessidade eminente de maior atenção por parte dos profissionais para esta problemática. Destaca-se, a importância da educação em saúde como ferramenta necessária para a garantia do autocuidado do paciente. Para assegurar um resultado efetivo, é fundamental que a equipe multiprofissional, e aqui se destaca o papel do enfermeiro, esteja qualificada para atuar junto ao paciente e família, nos processos que envolvem tratamento e estabelecimento de metas de controle e aquisição de novos comportamentos ${ }^{(10)}$.

O desconhecimento dos pacientes e a ausência de estratégias de educação em saúde aumentam a probabilidade do descontrole da doença e, por conseguinte, das complicações advindas em função desta. Dentre essas alterações estão às específicas do pé diabético, sendo considerada uma alteração de fisiopatologia complexa e de prevalência elevada, dependendo para sua prevenção e controle, de ações de saúde a priori simples baseadas na educação e interações multidisciplinares ${ }^{(17)}$.
Neste contexto, estudo recente sobre os cuidados de enfermagem ao paciente com pé diabético, elucida o papel fundamental do enfermeiro no desenvolvimento constante e permanente de atividades de educação em saúde propiciando desenvolver um vínculo de confiança com o usuário e qualificando o cuidado com o pé diabético. Por conseguinte, é importante que o enfermeiro busque envolver toda a equipe de saúde na busca e monitoramento dos fatores de risco e também no planejamento de ações como autocuidado e controle glicêmico para pessoas com a doençą ${ }^{(18)}$.

O sobrepeso, dislipidemia e hipertensão arterial associados à falta de controle glicêmico constituem-se como fatores de risco para o aparecimento de lesões em pés-diabéticos. Reitera-se que uma anamnese detalhada do enfermeiro incluindo aferição dos pulsos distais, teste de sensibilidade e orientações de prevenção são ferramentas importantes para o acompanhamento de pacientes com DM ${ }^{(19)}$.

Em relação aos cuidados específicos com os pés, foi possível evidenciar que os pacientes possuem práticas inadequadas, sobretudo quanto ao uso de calçados e aquisição dos mesmos, hidratação incorreta dos pés e corte inadequado das unhas. Sabe-se, entretanto, que o corte adequado das unhas é umas das orientações que o paciente diabético deve receber uma vez que o corte inadequado predispõe um quadro de unha encravada e consequentemente, aumenta a predisposição a infecções e lesões ${ }^{(15)}$.

Em uma pesquisa realizada sobre o cuidado diário com o pé diabético revelou que do total de 39 participantes do estudo, $28 \%$ afirmou que nunca observa a região plantar dos pés e $15 \%$ não usam lavar os pés diariamente. Assim, afirma que empoderar a pessoa com diabetes mellitus disponibilizando informações e conhecimentos pode aumentar a sua compreensão acerca da importância do cuidado com o pé diariamente pode favorecer a qualidade de vida e evitar possíveis lesões desnecessárias ${ }^{(20)}$. Neste estudo, os achados relacionados aos cuidados com os pés evidenciam a falta de empoderamento para este fazer, mostrando claramente a necessidade de educação em saúde para adoção de melhoras práticas de autocuidado.

Além de uma anamnese detalhada, é importante que o enfermeiro desenvolva habilidades e saiba reconhecer sinais e sintomas prévios que possam indicar diagnóstico precoce de neuropatia. No estudo em questão, os pacientes mencionaram, em sua maioria, sinais evidentes de dor, câimbras, dormência e formigamento. A neuropatia pode se manifestar ainda sob a forma de ausência de queixas, sendo erroneamente percebidas em situações de dormência e perda de sensibilidade (hipoestesia). Já sintomas de dor do tipo câimbra ou peso ao caminhar, que é aliviada ao repouso, pode relacionar-se à dor isquêmica por doença vascular periférica ${ }^{(16)}$. 
Reforça-se aqui o desafio maior da assistência de enfermagem, que consiste não só na capacitação dos profissionais para saberem identificar, avaliar e cuidar de pessoas com diabetes, mas também de favorecer o autocuidado e a adesão do tratamento por parte dos pacientes.

Os limites deste estudo estão relacionados ao tamanho da amostra. Possivelmente, com acesso a exames de imagem e laboratoriais as conclusões clínicas estariam mais fundamentadas acerca da doença neuropática e doença vascular periféricas como causa primária das características detectadas na avaliação dos pés. Apesar destes fatores limitadores considera-se a potencial contribuição do estudo para promoção da discussão acerca de ações aparentemente simples, mas com repercussões substanciais para a qualidade de vida das pessoas com diabetes, trata-se do acesso à informação e da construção de vínculos efetivos com os serviços e profissionais de saúde.

\section{CONCLUSÃO}

Os resultados deste estudo evidenciam que grande parte dos pacientes, apesar de terem o diagnóstico da doença por longo tempo, ainda desconhecem cuidados essenciais com os pés, realizam práticas inadequadas e raramente recebem orientações específicas para prevenir e evitar lesões nos pés. Ressalta-se assim, a lacuna existente nos serviços de saúde, quanto a ações educativas específicas para atender essa demanda de cuidado, sendo imperativo que os enfermeiros se apropriem desse conhecimento de modo que possam atuar de forma efetiva no cuidado aos pacientes diabéticos, minimizando lesões e internações futuras.

Considera-se a importância de novos estudos em diferentes contextos que corroborem com a construção do conhecimento acerca da temática com evidencias sobre as principais demandas de cuidado às pessoas com diabetes. 


\section{REFERÊNCIAS}

I. Pedrosa HC. Neuropatia dia ética periférica [Internet]. In: Tambascia M, Minicucci WJ, Pimazoni Netto A [Editores]. Sociedade Brasileira de Diabetes. E-Book 2.0 Diabetes na prática Clínica; 2015 [acesso em 21 jan 2020]. Disponível em: https://ebook.diabetes.org.br/main-page

2. Zhang P, Lu J, Jing Y,Tang S, Zhu D, Bi Y. Global epidemiology of diabetic foot ulceration: a systematic review and metaanalysis.Ann Med [Internet]. 2017 [acesso em 21 jan 2020]; 49:106-16. Disponível em: https://www.tandfonline.com/ doi/full/I0.1080/07853890.2016.1231932

3. Lazzarini PA, Hurn SE, Fernando ME, Scott D J, Kuys SS, Kamp MC, et al. Prevalence of foot disease and risk factors in general inpatient populations: a systematic review and meta-analysis. BMJ Open [Internet]. 2015 [acesso em 21 jan 2020]; 5:e008544. Disponível em: http://dx.doi.org/ I0.1 |36/ bmjopen-20I5-008544

4. Mishra SC, Chhatbar KC, Kashikar A, Mehndiratta Abha. Diabetic foot. BMJ [Internet]. 2017 [acesso em 21 jan 2020]; 359: j5064. Disponível em: https://www.bmj.com/ content/359/bmj.j5064.long

5. Toscano, Sugita, Rosa, Pedrosa, Rosa, Bahia. Annual Direct Medical Costs of Diabetic Foot Disease in Brazil: A Cost of Illness Study.Int. J. Environ. Rev. Public Health [Internet]. 2018 [acesso em 2I jan 2020];I5(I): 89. Disponível em: https://www.mdpi.com/l660-460I/I5/I/89/htm

6. Dorresteijn JAN, Valk GD. Patient education for preventing diabetic foot ulceration. Diabetes Metab Res Rev [Internet]. 2012 [acesso em I 3 mar 2020]; 28(Suppl I): I0 I-6. Disponível em:https://onlinelibrary.wiley.com/doi/ full/I0.1002/dmrr.2237

7. Bus SA, van Netten JJ, Lavery LA, Mnteiro-Soares M, Rasmussen A, Jubiz Y, et al. International Working Group on the Diabetic Foot guidance on the prevention of foot ulcers in at-risk patients with diabetes. Diabetes Metab Res Rev [Internet]. 2016 [acesso em 13 mar 2020]; 32: I624. Disponível em: https://onlinelibrary.wiley.com/doi/ full/I0.1002/dmrr.2696

8. Padilha AP, Rosa LM, SchoellerSS, JunkesC, MendezCB, Martins MMFPS. Manual de cuidados às pessoas com diabetes e pé diabético: construção por scopingstudy. Texto \& contexto enferm [Internet].2017 [acesso em I 3 mar 2020]; 26(4):e2 1900 I7. Disponível em:http://www.scielo.br/scielo. php?script=sci_arttext\&pid=SO I04-070720 I 7000400322

9. International Diabetes Federation. IDF Diabetes Atlas [Internet]. 8th ed. Brussels, Belgium: International Diabetes Federation; 2017 [acesso em 02 ago 2019]. Disponível em: https://www.idf.org/e-library/ epidemiology-research/diabetes-atlas/ / 34-idf-diabetesatlas-8th-edition.html

10. Sociedade Brasileira de Diabetes. Diretrizes da Sociedade Brasileira de Diabetes 2017-2018 [Internet]. São Paulo: Editora Clannad; 2017 [acesso em 02 ago 2019]. Disponível em: https://www.diabetes.org.br/profissionais/images/2017/ diretrizes/diretrizes-sbd-2017-2018.pdf

II. Instituto Brasileiro de Geografia e Estatística. Pesquisa Nacional de Saúde 2013: percepção do estado de saúde, estilos de vida e doenças crônicas. Brasil, grandes regiões e unidades da federação [Internet]. Rio de Janeiro:IBGE; 20I4 [acesso em 02 ago 2019]. Disponível em: https://biblioteca. ibge.gov.br/visualizacao/livros/liv94074.pdf

12. Gomes LC, Silva Junior AJ. Fatores favoráveis ao pé diabético em usuários de uma unidade de atenção primária à saúde. Rev. Aten. Saúde [Internet]. 2018 [acesso em 13 mar 2020]; I6(57):5-12. Disponível em: https://seer.uscs.edu.br/ index.php/revista_ciencias_saude/article/view/4943/pdf_I

13. Lima IG, Costa JFL, Oliveira AF, Borges Junior JN, Peixoto AS, Pancieri MS, et al. Educar para prevenir: a importância da educação no cuidado do pé diabético. Rev. Conexao UEPG [Internet]. 2017 [acesso em 13 mar 2020]; I3(I): 186-95. Disponível em: https://revistas.apps.uepg.br/index. $\mathrm{php/conexao/article/view/8958/550I}$

14. Cubas MR, dos Santos OM, Retzlaff EMA, Telma HLC, de Andrade IPS, Moser ADL, et al. Pé diabético: orientações e conhecimento sobre cuidados preventivos. Fisioter. Mov. [Internet]. 2013 [acesso em I 3 mar 2020]; 26(3): 647-55. Disponível em: http://www.scielo.br/pdf/fm/v26n3/ a 19v26n3.pd

15. Ministério da Saúde (BR). Secretaria de Atenção à Saúde. Departamento de Atenção Básica. Manual do pé diabético: estratégias para o cuidado da pessoa com doença crônica. Brasília: Ministério da Saúde; 2016.

16. Santos AG. Mapeando pés: prevenir lesões nos pés de utentes com diabetes tipo 2 [dissertação]. Setúbal (Portugal): Escola Superior de Saúde - Instituto Politécnico de Setúbal; 2019.

17. Caiafa JS, Castro AA, Fidelis C, Santos VP, Silva ES, Sitrângulo Junior CJ. Atenção integral ao portador de Pé Diabético. J Vasc Bras [Internet]. 201 I [acesso em I 3 mar 2020]; 10(4) Supl. 2: I-32. Disponível em: http://www.scielo.br/pdf/jvb/ vI0n4s2/a0lv10n4s2

18. Silva Filho JP, Andrade SG, Lima TFS, Name KPO. Os cuidados de enfermagem junto ao paciente com o pé diabético. ReBIS [Internet]. 2019 [acesso em I 3 mar 2020]; I(3):6-I I. Disponível em: https://revista.rebis.com.br/index. php/rebis/article/view/I /4/38

19. Zagury L. Tratamento Atual do Diabetes mellitus. Rio de janeiro: Guanabara Koogan; 2010.

20. Santos CRV, Carvalho EF, Souza WV, Albuquerque EC. Fatores associados a amputações por pé diabético. J Vasc Bras [Internet]. 2015 [acesso em 13 mar 2020]; I4(I):3745. Disponível em: http://www.scielo.br/pdf/jvb/vl4nI/ pt_I677-5449-jvb-|4-0I-00037.pdf

Recebido: 2020-04-02 Aceito: 2020-05-22 